\title{
Latest Developments for In Situ Measurements of Gas-Solid Interactions
}

\author{
Renu Sharma, ${ }^{1}$ Pin Ann Lin, ${ }^{1,2}$ Matthieu Picher, ${ }^{1,2}$ Zahra Hussaini ${ }^{1}$ \\ ${ }^{1 .}$ Center for Nanoscale Science and Technology, National Institute of Standards and Technology, \\ Gaithersburg, MD 20899-6203; \\ 2. Institute for Research in Electronics and Applied Physics, University of Maryland, College park, MD \\ 20740
}

The power of in situ observations at elevated temperatures to elucidate the details of gas-solid interactions was realized during the very early years of the advent of transmission electron microscope [1]. Of several designs developed between 1950 and 1970, the underlying approach of using a differential pumping system, as proposed by Swann and Tighe [2], has been further developed and is now widely used in commercially-available environmental transmission or scanning transmission electron microscopes (ETEM or ESTEM). In recent years ESTEM, has been successfully employed to reveal and understand the structural and chemical changes occurring in nanoparticles under reactive environments [3,4]. However, quantitative measurements of reaction rates and chemical changes are precluded by (a) the nanoscale region used for atomic imaging, (b) uncertainties in the actual temperature of the region under observation, and (c) the large amount of data to be analyzed. Here we present various technical and analytical methods we have developed to address these issues.

First, we have incorporated a free-standing, broad-band light-focusing system in the ESTEM to excite and collect vibrational and optical spectra from TEM samples under reactive environments.[5] We have used it collect Raman spectra during the in situ growth of single-walled carbon nanotubes (SWCNTs) from $\approx 80 \mu \mathrm{m}^{2}$ area $\left(\approx 10 \mu \mathrm{m}\right.$ diameter laser beam) while collecting atomic scale images from a $200 \mathrm{~nm}^{2}$ area at $625^{\circ} \mathrm{C}$ in $0.005 \mathrm{~Pa}$ of $\mathrm{C}_{2} \mathrm{H}_{2}$. Figure 1 shows the growth kinetics as measured (a) from the change in intensity of the G band of the SWCNTs in the Raman spectra and (b) of an individual tube from high resolution images extracted from a video sequence. It is important to note the overall shape of the growth curve and growth regimes are comparable for the two measurements, confirming that atomicscale data for this reaction is representative of the entire sample.

The shifts in the Raman peaks with increasing temperature were also used to calibrate the sample temperature. For a membrane heating chip, we find that the temperature drops as we move away from the central hole (Fig. 2a). Moreover, the temperature drop due to introduction of $\mathrm{N}_{2}$ was also measured as a function of pressure for different temperatures (Fig. 2b).

In order to obtain quantitative information from the large number of images generated during in situ measurements, we have developed a scheme that utilizes a combination of home-built and publicallyavailable algorithms for image drift correction, noise reduction and template matching to accurately locate the position of atomic columns. The latter employs a Delaunay triangulation to connect each point to its nearest neighbors, after which, the average nearest neighbor distance for each point is calculated. For each triangle, a value of the local spacing is calculated as the average of the average nearest neighbor distances of its three points to determine the structure. Fig. 3 shows the fluctuation between the $\mathrm{Co}$ and $\mathrm{Co}_{2} \mathrm{C}$ area fraction during the SWCNT growth. A detailed description of the techniques and their applications will be presented.

References:

[1] Butler and Hale, (1981) Practical Methods in electron microscopy, Elsevier/North Holland Co.

[2] Swann and Tighe, Proc. $5^{\text {th }}$ Eur. Reg. Cong., p. 436 
[3] Sharma, R., J. Mat. Res. 20 (2005), p. 1695.

[4] Hansen et al, Science 294 (2001), p. 1508.

[5] Picher et al, Ultramicroscopy 150 (2015), p. 10.

Figure 1. A: SWCNT growth rate measured from the evolution of $\mathrm{G}$ band in Raman signal. B: linear growth rate of an individual

SWCNT measured from atomicresolution video data. Note the similarity in the growth curves.
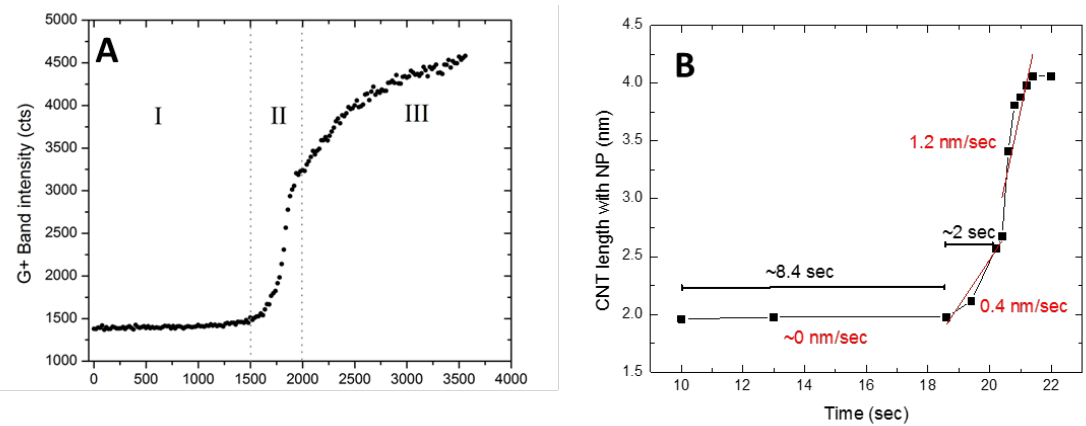

Figure 2. a) Temperature variations measured from the Raman shifts away from the center of a membrane heating chip. b) Temperature drop with increasing $\mathrm{N}_{2}$ gas pressure at different temperatures. Note the advent of the drop is dependent on both the pressure and initial temperature.
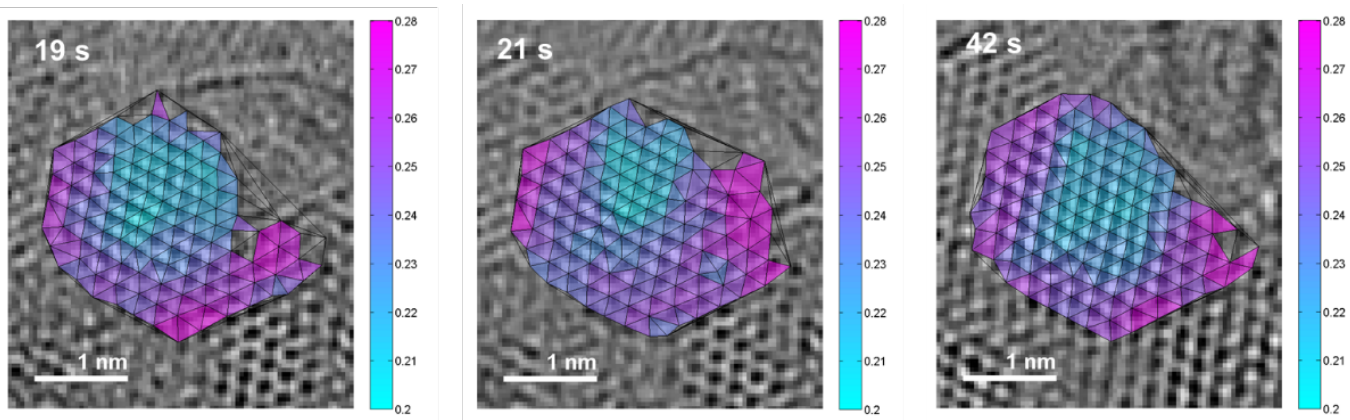

Figure 3. Fluctuation in the size of $\mathrm{Co}$ (marked blue) and $\mathrm{Co}_{2} \mathrm{C}$ (marked violet) areas during the growth of SWCNT as measured using the image analysis method described in the text. The time is given at the right hand corner. These modulations can be directly related to the growth rate of SWCNT 